\title{
A Class of Two-Derivative Two-Step Runge-Kutta Methods for Non-Stiff ODEs
}

\section{B. Aiguobasimwin and R. I. Okuonghae $\mathbb{D}$}

Department of Mathematics, University of Benin, Nigeria

Correspondence should be addressed to R. I. Okuonghae; okunoghae01@yahoo.co.uk

Received 23 December 2018; Accepted 30 April 2019; Published 20 May 2019

Academic Editor: Igor Andrianov

Copyright (C) 2019 I. B. Aiguobasimwin and R. I. Okuonghae. This is an open access article distributed under the Creative Commons Attribution License, which permits unrestricted use, distribution, and reproduction in any medium, provided the original work is properly cited.

In this paper, a new class of two-derivative two-step Runge-Kutta (TDTSRK) methods for the numerical solution of non-stiff initial value problems (IVPs) in ordinary differential equation (ODEs) is considered. The TDTSRK methods are a special case of multiderivative Runge-Kutta methods proposed by Kastlunger and Wanner (1972). The methods considered herein incorporate only the first and second derivatives terms of ODEs. These methods possess large interval of stability when compared with other existing methods in the literature. The experiments have been performed on standard problems, and comparisons were made with some standard explicit Runge-Kutta methods in the literature.

\section{Introduction}

In this paper we propose a class of the TDTSRK methods for the numerical solution of the IVPs in ODEs,

$$
y^{\prime}=f(y), \quad x \in\left[x_{0}, X\right], y\left(x_{0}\right)=y_{0},
$$

where $g=y^{\prime \prime}=f_{y} f$ and $f: \mathbb{R}^{N} \longrightarrow \mathbb{R}^{N}$ and $g: \mathbb{R}^{N} \longrightarrow$ $\mathbb{R}^{N}$. Examples of problems leading to ODEs (1) are in [1-18]. The new TDTSRK is

$$
\begin{aligned}
Y_{i}^{[n]}= & \left(1-u_{i}\right) y_{n-1}+u_{i} y_{n-2}+h \sum_{j=1}^{i-1} a_{i j} f\left(Y_{j}^{[n]}\right) \\
& +h \sum_{j=1}^{s} b_{i j} f\left(Y_{j}^{[n-1]}\right)+h^{2} \sum_{j=1}^{s} \bar{b}_{i j} g\left(Y_{j}^{[n-1]}\right), \\
y_{n}= & (1-\theta) y_{n-1}+\theta y_{n-2} \\
& +h \sum_{j=1}^{s}\left(v_{j} f\left(Y_{j}^{[n]}\right)+w_{j} f\left(Y_{j}^{[n-1]}\right)\right)
\end{aligned}
$$

$$
\begin{aligned}
+h^{2} \sum_{j=1}^{s}\left(\bar{v}_{j} g\left(Y_{j}^{[n]}\right)\right. & \left.+\bar{w}_{j} g\left(Y_{j}^{[n-1]}\right)\right), \\
& \theta \in[-1,1], n=2,3, \ldots, N .
\end{aligned}
$$

The $h$ is the step size and $c=\left[c_{1}, \cdots c_{s}\right]^{T}$ is the abscissa value. The abscissa value $\left\{c_{i}\right\}_{i=1}^{s}$ represents the positions of the the internal stages. The $y^{\prime \prime}(x)=g=f_{y} f$ is the second derivative form of ODEs (1). The $y_{n}$ is an approximation to the exact solution $y\left(x_{n}\right)$. The past and present stage values are $Y_{i}^{[n-1]}=y\left(x_{n-2}+c_{i} h\right)$ and $Y_{i}^{[n]}=y\left(x_{n-1}+c_{i} h\right)$ respectively, while the first and second derivatives of the past stage values are $f\left(Y_{j}^{[n-1]}\right)$, and $g\left(Y_{j}^{[n-1]}\right)$ respectively. The present stage value and its derivatives are

$$
Y^{[n]}=\left(\begin{array}{c}
Y_{1}^{[n]} \\
\vdots \\
Y_{s}^{[n]}
\end{array}\right) \text {, }
$$




$$
\begin{gathered}
F\left(Y^{[n]}\right)=\left(\begin{array}{c}
f\left(Y_{1}^{[n]}\right) \\
\vdots \\
f\left(Y_{s}^{[n]}\right)
\end{array}\right), \\
G\left(Y^{[n]}\right)=\left(\begin{array}{c}
g\left(Y_{1}^{[n]}\right) \\
\vdots \\
g\left(Y_{s}^{[n]}\right)
\end{array}\right) .
\end{gathered}
$$

$$
\begin{aligned}
& +h\left((A \otimes I) F\left(Y^{[n]}\right)+(B \otimes I) F\left(Y^{[n-1]}\right)\right) \\
& +h^{2}(\bar{B} \otimes I) G\left(Y^{[n-1]}\right) \\
y_{n}= & (1-\theta) y_{n-1}+\theta y_{n-2} \\
& +h\left((V \otimes I) F\left(Y^{[n]}\right)+(W \otimes I) F\left(Y^{[n-1]}\right)\right) \\
& +h^{2}\left((\bar{V} \otimes I) G\left(Y^{[n]}\right)+(\bar{W} \otimes I) G\left(Y^{[n-1]}\right)\right),
\end{aligned}
$$

The compact form of (2) is

$$
Y^{[n]}=((e-u) \otimes I) y_{n-1}+(u \otimes I) y_{n-2}
$$

(3)

where $e=[1,1 \ldots, 1]^{T} \in R^{s}$ and $c=\left[c_{1}, \ldots, c_{s}\right]^{T}, A=\left[a_{i j}\right]$, $U=\left[u_{i j}\right], \bar{A}=0, B=\left[b_{i j}\right], \bar{B}=\left[\bar{b}_{i j}\right], V=\left[v_{j}\right], W=\left[w_{j}\right]$, $\bar{V}^{T}=\left[\bar{v}_{j}\right], \bar{W}=\left[\bar{w}_{j}\right]$, and $\theta$ is a scalar and 0 is a null matrix. The Butcher tableau of the method in (2) is

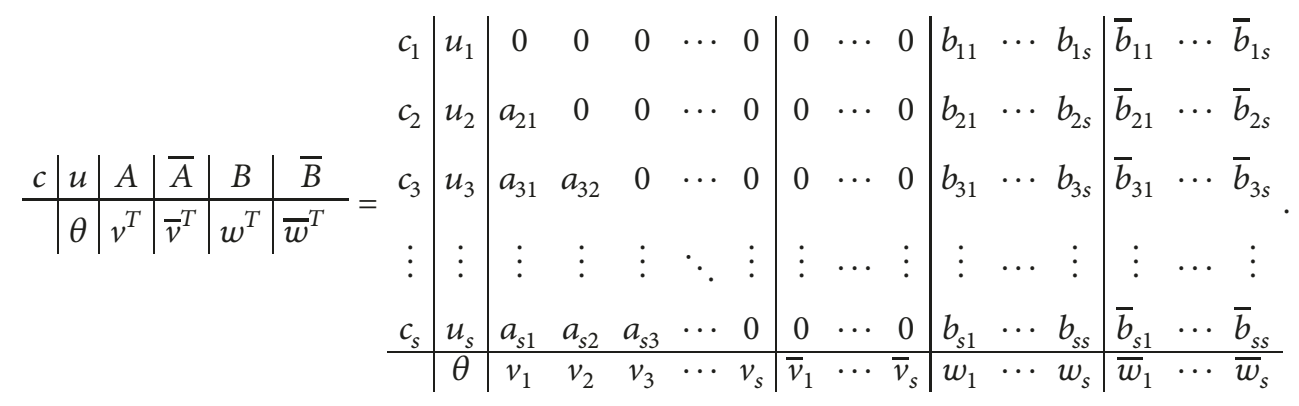

The $A$ is strictly lower trigular matrix while $\bar{A}$ is a null matrix. The method in (2) is an extension of the methods in [2$4,7,9-13,19]$, and a subclass of the methods in $[3,5,8,15$, 17]. Some of advantages of the TDTSRK methods over the classical Runge-Kutta (RK) methods have been highlighted in $[5,8,15,17]$. In Section 2 , the order conditions and the stability analysis of the TDTSRK methods are stated. In Section 3, we derive TDTSRK methods using the stated order conditions and in Section 5, numerical results are presented.

\section{The Order Condition and Stability Properties of the TDTSRK Methods}

Butcher and Tracogna [3] have shown that the order conditions for TSRK method of order $p$ can be tabulated conveniently using mapping of all rooted tress. In the spirit of [3], Turac and Ozi [18] obtained the order conditions of TDTSRK. Here, we use an equivalent set of order conditions, which may be solved directly using the strategies described in [19] and it follows from the results of [19] that the necessary and sufficient conditions which the method in (2) must satisfy in order to have methods of order $q$ and stage order $p$ are $h d\left(x_{n}\right)=\sum_{j}^{\infty} \widehat{C}_{j} y^{(j)}\left(x_{n-1}\right) h^{j}$ and $\widetilde{h d}\left(x_{n}\right)=$ $\sum_{j}^{\infty} C_{j} y^{(j)}\left(x_{n-1}\right) h^{j}$ with

$$
C=(A+B) e+\bar{B} e-u ; \quad j=1,
$$

$$
\begin{aligned}
& C_{j} \\
& =\frac{c^{j}}{j !}-\frac{(-1)^{j}}{j !} u-A \frac{c^{j-1}}{(j-1) !}-B \frac{(c-e)^{j-1}}{(j-1) !} \\
& \quad-\bar{B} \frac{(c-e)^{j-2}}{(j-2) !} ; j=2(1) q, \\
& \left(V^{T}+W^{T}\right) e+\left(\bar{V}^{T}+\bar{W}^{T}\right) e=1+\theta ; \quad j=1, \\
& \widehat{C}_{j} \quad \\
& =\frac{1}{j}-\frac{(-1)^{j}}{j !} \theta-V^{T} \frac{c^{j-1}}{(j-1) !}-\bar{V}^{T} \frac{c^{j-2}}{(j-2) !} \\
& \quad-W^{T} \frac{(c-e)^{j-1}}{(j-1) !}-\bar{W}^{T} \frac{(c-e)^{j-2}}{(j-2) !} ; j=2(1) p .
\end{aligned}
$$

We investigate the stability properties of the TDTSRK methods using the standard test equation:

$$
y^{\prime}=\lambda y
$$


where $\lambda$ is a complex parameter. Casting the method in (2) in general linear method (GLM) format gives

$$
\begin{aligned}
& \left(\begin{array}{c}
Y^{[n]} \\
y_{n} \\
y_{n-1} \\
h f\left(Y^{[n]}\right) \\
h^{2} g\left(Y^{[n]}\right)
\end{array}\right) \\
& =\left(\begin{array}{c|c|cccc}
\mathbf{0} & \overline{\mathbf{A}} & \mathbf{e}-\mathbf{u} & \mathbf{u} & \mathbf{B} & \overline{\mathbf{B}} \\
\hline \mathbf{v}^{T} & \overline{\mathbf{v}}^{T} & 1-\vartheta & 9 & \mathbf{w}^{T} & \overline{\mathbf{w}}^{T} \\
\mathbf{0} & \mathbf{0} & 1 & \mathbf{0} & \mathbf{0} & \mathbf{0} \\
\mathbf{I} & \mathbf{0} & \mathbf{0} & \mathbf{0} & \mathbf{0} & \mathbf{0} \\
\mathbf{0} & \mathbf{I} & \mathbf{0} & \mathbf{0} & \mathbf{0} & \mathbf{0}
\end{array}\right)\left(\begin{array}{c}
h f\left(Y^{[n]}\right) \\
\frac{h^{2} g\left(Y^{[n]}\right)}{y_{n-1}} \\
y_{n-2} \\
h f\left(Y^{[n-1]}\right) \\
h^{2} g\left(Y^{[n-1]}\right)
\end{array}\right) .
\end{aligned}
$$

Applying the GLM in (7b) to (7) yields

$$
M(z)=P+\left(z V+z^{2} \bar{V}\right)\left(I-z^{2} \bar{A}\right)^{-1} \Theta
$$

where $M(z)$ is the stability matrix, $\Theta=(\mathbf{e}-\mathbf{u} \quad \mathbf{u} \quad \mathbf{B} \quad \overline{\mathbf{B}})$ and

$$
\begin{aligned}
P & =\left(\begin{array}{cccc}
1-\vartheta & \vartheta & \mathbf{w}^{T} & \overline{\mathbf{w}}^{T} \\
1 & \mathbf{0} & \mathbf{0} & \mathbf{0} \\
\mathbf{0} & \mathbf{0} & \mathbf{0} & \mathbf{0} \\
\mathbf{0} & \mathbf{0} & \mathbf{0} & \mathbf{0}
\end{array}\right) ; \\
V & =\left(\begin{array}{c}
v^{T} \\
\mathbf{0} \\
\mathbf{I} \\
\mathbf{0}
\end{array}\right) ; \\
\bar{V} & =\left(\begin{array}{c}
\bar{v}^{T} \\
\mathbf{0} \\
\mathbf{0} \\
\mathbf{I}
\end{array}\right)
\end{aligned}
$$

The stability polynomial, $p(w, z)$ of the GLM in $(7 b)$ is

$$
p(w, z)=\operatorname{det}(w I-M(z))
$$

where $I$ is the identity matrix of the same order with matrix $M(z)$.

\section{The TDTSRK Methods with Large Region of Absolute Stability}

In this section, we consider methods with stage $s=1$, $s=2$ and $s=3$ of order two, four, and six, respectively. In (2), $A$ is strictly lower triangular matrix while $\bar{A}$ is null matrix.
3.1. TDTSRK Method of Order $p=2, q=2, s=1$. In the spirit of $[11,19]$ we obtain the coefficients of TDTSRK method of order $p=2$ as

$$
\begin{aligned}
& b_{11}=c_{1}+u_{1}, \\
& \bar{b}_{11}=\frac{1}{2}\left(2 c_{1}-c_{1}^{2}+u_{1}-2 c_{1} u_{1}\right), \\
& w_{1}=1-v_{1}, \\
& \bar{w}_{1}=\frac{1}{2}\left(3-2 c_{1}-2 \bar{v}_{1}-2 v_{1}\right) .
\end{aligned}
$$

The tableau of the method of order $p=2$ is

$$
\begin{aligned}
& \begin{array}{c|c|c|c|c|c}
c & u & A & 0 & B & \bar{B} \\
\hline & \theta & v^{T} & \bar{v}^{T} & w^{T} & \bar{w}^{T}
\end{array} \\
& =\begin{array}{c|c|c|c|c|c}
1 & \frac{25}{26} & 0 & 0 & \frac{51}{26} & \frac{1}{52} \\
\hline 0 & \frac{-14}{40} & \frac{-2}{25} & \frac{27}{20} & \frac{127}{140}
\end{array} .
\end{aligned}
$$

The stability polynomial of this method is

$$
\begin{aligned}
p(w, z)= & w^{4}-w^{3}-p_{3}(z) w^{3}+p_{2}(z) w^{2} \\
& +p_{1}(z) w
\end{aligned}
$$

where

$$
\begin{aligned}
& p_{3}(z)=\frac{-1013 z}{520}-\frac{\left(31 z^{2}\right)}{1820}, \\
& p_{2}(z)=\frac{146 z}{65}+\frac{11 z^{2}}{280}, \\
& p_{1}(z)=\frac{-135 z}{104}-\frac{\left(635 z^{2}\right)}{728} .
\end{aligned}
$$

The interval of absolute stability is approximately equal to $[-5.53,0]$.

3.2. TDTSRK Method of Order $p=4, q=2, s=2$. Fixing $p=4$ and solving the arising system of order conditions and stage order conditions $C_{j}=0, j=1(1) 3$ and $\widehat{C}_{j}=0, j=1,2$ with respect to $b_{11}, b_{21}, b_{11}, \bar{b}_{21}, w_{1}, w_{2}$ and $\bar{w}_{1}$ in (6) we obtain a twelve-parameter family of methods of order $p=4$ and 
stage order $q=2$. The coefficients of the methods are given by

$$
\begin{aligned}
& b_{11}=-b_{12}+u_{1}, \\
& \bar{b}_{11}=-b_{12}-\bar{b}_{12}+\frac{u_{1}}{2}, \\
& b_{21}=1-a_{21}-b_{22}+u_{2}, \\
& \bar{b}_{21}=\frac{3}{2}-a_{21}-b_{22}-\bar{b}_{22}+\frac{u_{2}}{2}, \\
& w_{1}=\frac{3}{2}-12 \bar{v}_{2}-5 v_{2}, \\
& w_{2}=\frac{-1}{2}+12 \bar{v}_{2}-v_{1}+4 v_{2}, \\
& \bar{w}_{1}=\frac{7}{12}-5 \bar{v}_{2}-2 v_{2}, \\
& \bar{w}_{2}=\frac{17}{12}-\bar{v}_{1}-8 \bar{v}_{2}-4 v_{2} .
\end{aligned}
$$

The coefficients of the resulting TDTSRK method are

$$
\begin{aligned}
& \begin{array}{c|c|c|c|c|c}
c & u & A & 0 & B & \bar{B} \\
\hline & \theta & v^{T} & \bar{v}^{T} & w^{T} & \bar{w}^{T}
\end{array} \\
& =\begin{array}{c|c|cc|cc|cc|cc}
0 & \frac{1}{9} & 0 & 0 & 0 & 0 & \frac{-52}{99} & \frac{7}{11} & \frac{-707}{990} & \frac{2}{15} \\
1 & \frac{1}{15} & \frac{17}{26} & 0 & 0 & 0 & \frac{601}{4290} & \frac{3}{11} & \frac{-371}{8580} & \frac{13}{20} \\
\hline & 0 & \frac{1}{9} & \frac{1}{6} & \frac{-1}{10} & \frac{-1}{8} & \frac{13}{6} & \frac{-13}{9} & \frac{7}{8} & \frac{37}{20}
\end{array}
\end{aligned}
$$

The stability polynomial of this method takes the form

$$
\begin{aligned}
p(w, z)= & w^{6}-w^{5}-p_{5}(z) w^{5}+p_{4}(z) w^{4} \\
& +p_{3}(z) w^{3}+p_{2}(z) w^{2}
\end{aligned}
$$

where

$$
\begin{aligned}
& p_{5}(z)=-\frac{8 z}{4455}-\frac{4696 z^{2}}{19305}-\frac{17 z^{3}}{1170}, \\
& p_{4}(z)=-\frac{7607 z}{8910}-\frac{3890911 z^{2}}{231660}-\frac{1043 z^{3}}{720}-\frac{24877 z^{4}}{58500}, \\
& p_{3}(z)=-\frac{13 z}{90}+\frac{18021 z^{2}}{772200}+\frac{3914887 z^{3}}{4633200}-\frac{528301 z^{4}}{1716000}, \\
& p_{2}(z)=\frac{139 z^{2}}{2970}+\frac{21977 z^{3}}{231660}-\frac{122381 z^{4}}{5148000} .
\end{aligned}
$$

The interval of absolute stability of the fourth-order TDTSRK method is approximately equal to $[-14.68,0]$.

3.3. TDTSRK Method of Order $p=6, q=4, s=3$. We conclude this section with the construction of TDTSRK methods of order $p=6$ and stage order $q=4$. Solving the system of order and stage order condition $C_{j}$, where $j=1(1) 6$ and $\widehat{C}_{j}=0$ where $j=1(1) 4$ with respect to $b_{11}, \bar{b}_{11}, b_{12}, \bar{b}_{12}$, $b_{21}, \bar{b}_{21}, b_{22}, \bar{b}_{22}, b_{31}, \bar{b}_{31}, b_{32}, \bar{b}_{32}, w_{1}, w_{2}, w_{3}, \bar{w}_{1}, \bar{w}_{2}$ and $\bar{w}_{3}$, we obtain a twenty-one-parameter family of methods of order $p=6$ and stage order $q=4$; see appendix for the coefficients of TDTSRK methods of order $p=6$. The Butcher's tableau is

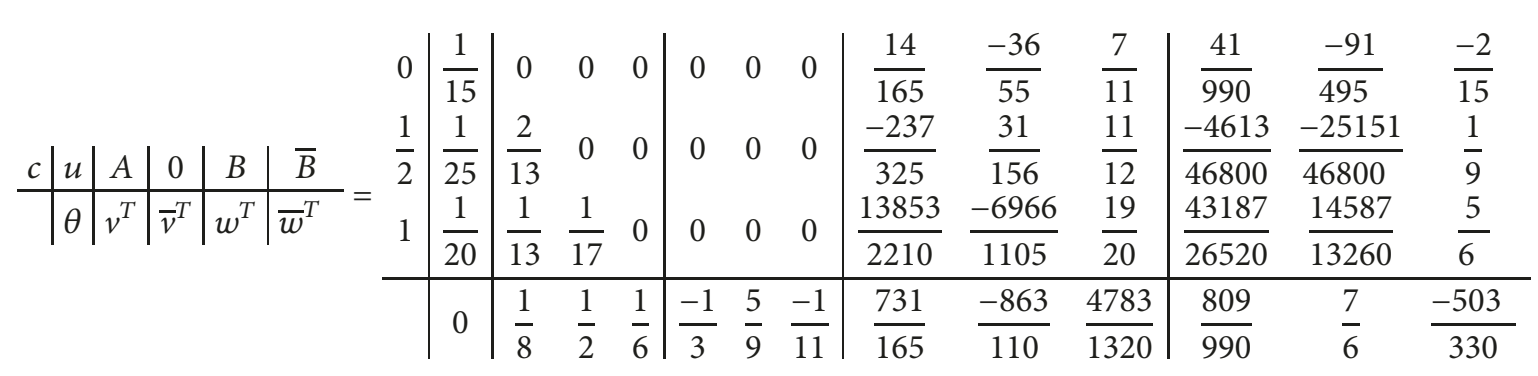

The stability polynomial of this method is

$$
\begin{aligned}
p(w, z)= & w^{8}-w^{7}-p_{7}(z) w^{7}+p_{6}(z) w^{6} \\
& +p_{5}(z) w^{5}+p_{4}(z) w^{4}+p_{3}(z) w^{3},
\end{aligned}
$$

where

$$
\begin{aligned}
& p_{7}(z)=\frac{-56873 z}{28600}-\frac{97561 z^{2}}{171600}-\frac{71011 z^{3}}{1640925}+\frac{24 z^{4}}{12155} \\
& p_{6}(z)=\frac{5926769 z}{5148000}+\frac{730039487 z^{2}}{262548000}-\frac{852286207 z^{3}}{3850704000}
\end{aligned}
$$

$$
\begin{aligned}
& -\frac{632475763 z^{4}}{3057912000}+\frac{12819901 z^{5}}{147683250}, \\
p_{5}(z)= & -\frac{64433 z}{396000}-\frac{25121949287 z^{2}}{19253520000} \\
& -\frac{89071610501 z^{3}}{9241689600}+\frac{2262450564601 z^{4}}{693126720000} \\
& +\frac{34222229413 z^{5}}{519845040000}-\frac{427138145153 z^{6}}{1559535120000}, \\
p_{4}(z)= & -\frac{33582133259 z^{2}}{57760560000}+\frac{1662083701181 z^{3}}{231042240000}
\end{aligned}
$$




$$
\begin{aligned}
& +\frac{1341594268543 z^{4}}{259922520000}+\frac{301196593151 z^{5}}{319904640000} \\
& +\frac{260210961613 z^{6}}{1039690080000} \\
p_{3}(z)= & -\frac{1729648873 z^{3}}{28600}+\frac{87855429059 z^{4}}{173281680000} \\
& +\frac{170828953577 z^{5}}{1039690080000}+\frac{43438448533 z^{6}}{779767560000} .
\end{aligned}
$$

The interval of absolute stability of the sixth order TDTSRK method is approximately equal to $[-30.24,0]$. See Figure 1.

The stability plots of TDTSRK (2) in Figure 1 show that the interval of absolute stability of the method in (2) is larger than that of the TDTSRK methods in RK [4], TDRK [5], and TSRK $[10,18]$. This serves as an advantage over other existing methods and this justifies the inclusion of the second derivative term of the ODEs (1) and the generalization of TSRK method in [10].

\section{Numerical Experiment}

In this section, we solve some initial value problems, and our results were compared with the results of other researchers in the literature. The fourth-order methods used for comparing are as follows: (i). TDRK methods in [5], (ii). SDTSRK methods in [15], (iii). ESDTSRK methods in [20], (iv). TDTSRK methods (new) in Section 3.2, and (v). TDTSRK methods in [18].

To start up the algorithms in (i)-(v) we use the initial value $y_{0}$ and compute the value of $y_{1}$ from the exact solution or onestep explicit method of order four. For easy implementation, the methods in (i)-(v) are designed to have the capacity of varying step size. Variable stepsize strategy proposed in [4] is applied herein. The non-stiff and mildly stiff problems solved are as follows:

Problem 1. The electrical circuit problem [21],

$$
\begin{aligned}
& y_{1}^{\prime}(x)=-4 y_{1}+3 y_{2}+6, \\
& y_{1}(0)=0, \\
& y_{1}(x)=-3.375 e^{-2 x}+1.875 e^{-0.4 x}+1.5, \\
& y_{2}^{\prime}(x)=-2.4 y_{1}+1.6 y_{2}+3.6, \\
& y_{2}(0)=0, \\
& y_{2}(x)=-2.25 e^{-2 x}+2.25 e^{-0.4 x},
\end{aligned}
$$

Problem 2. Nonlinear chemical problem

$$
\begin{aligned}
& y_{1}^{\prime}(x)=-y_{1}, \\
& y_{1}(0)=1, \\
& y_{2}^{\prime}(x)=y_{1}-y_{2}^{2}, \\
& y_{2}(0)=0, \\
& y_{3}^{\prime}(x)=y_{2}^{2}, \\
& y_{3}(0)=0,
\end{aligned}
$$

$$
x=[0,5] .
$$

Problem 3. The Kaps problem (see $[5,18])$

$$
\begin{aligned}
& y_{1}^{\prime}(x)=-y_{1}\left(1+y_{1}\right)+y_{2}, \\
& y_{1}(0)=1, \\
& y_{1}(x)=e^{-x} \\
& y_{2}^{\prime}(x)=\tau\left(y_{1}^{2}-y_{2}\right)-2 y_{2}, \\
& y_{2}(0)=1, \\
& y_{2}(x)=e^{-2 x}
\end{aligned}
$$

$$
x=[0,1] .
$$

This problem is mildly stiff. We take $\tau=100$ and the results are given in Figure 4. The third-order error estimator formula for the methods in (i)-(v) is

$$
\begin{aligned}
\bar{y}_{n} & =y_{n-1}+h\left(\frac{1}{11} f\left(Y_{1}^{[n]}\right)+\frac{1}{22} f\left(Y_{2}^{[n]}\right)\right. \\
& \left.-\frac{13}{66} f\left(Y_{1}^{[n-1]}\right)+\frac{35}{33} f\left(Y_{2}^{[n-1]}\right)\right)+h^{2}\left(\frac{1}{2} g\left(Y_{1}^{[n]}\right)\right. \\
& \left.-\frac{8}{33} g\left(Y_{2}^{[n-1]}\right)\right), \quad c=[0,1]^{T}, p=3 .
\end{aligned}
$$

The error estimators for order $p=2$ and $p=6$ in this paper are given in [20]. In Figures 2(a), 2(b), 3(a), 3(b), 4(a), and 4(b), the notations used are as follows:

TOL: Tolerance used, $n f e$ : number of function evaluations, CPU time: is the computational time and is measured in seconds,

$\left\|\bar{y}_{n}-y_{n}\right\|_{\infty}$ : Is the maximum error and is obtained from the difference between error estimate formula, $\bar{y}_{n}$ and the output method, $y_{n}$ of TDTSRK method in Section 3.2,

$\left\|y_{\text {exact }}-y_{n}\right\|_{\infty}$ : is the maximum global error and is the difference between the exact and the numerical solution, where $y_{\text {exact }}$ and $y_{n}$ are the exact and the numerical solutions, respectively.

The tests compare five different fourth-order methods with three different tolerances, Tol $=10^{-j}, j=1,2,3$ for Problems 1-3 and for every tolerance the same initial step size is used. When carrying out a comparison among numerical methods, the criterion to be used is very important. So, if 


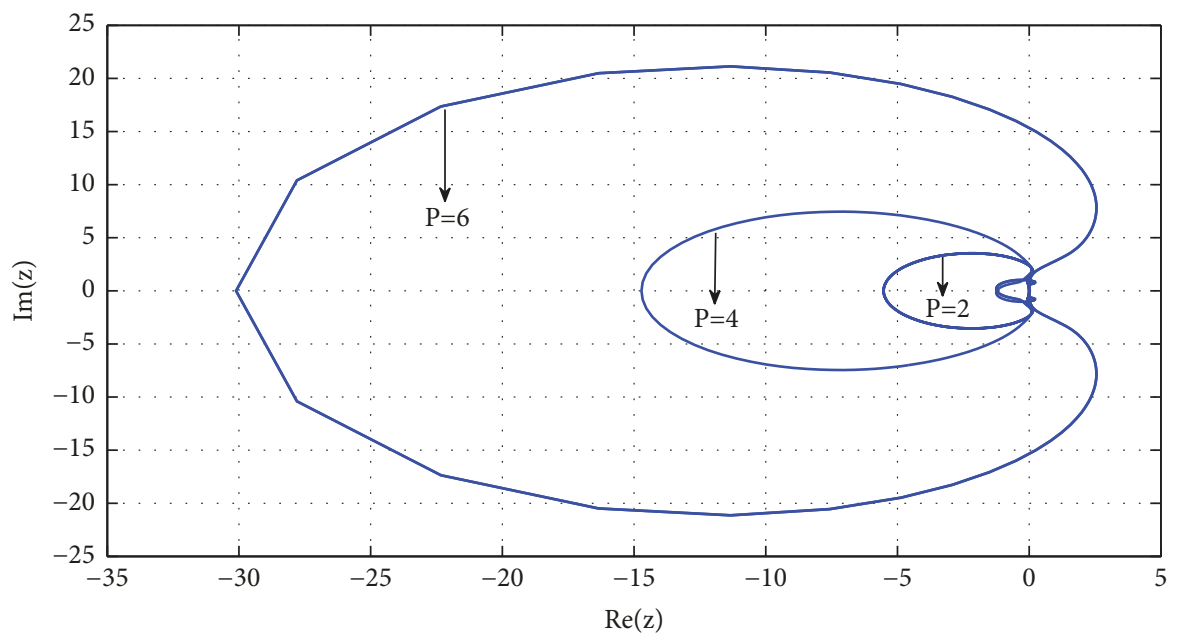

FIGURE 1: The region of absolute stability (closed curve) of TDTSRK methods in (7) for order $p=2, p=4$, and $p=6$.

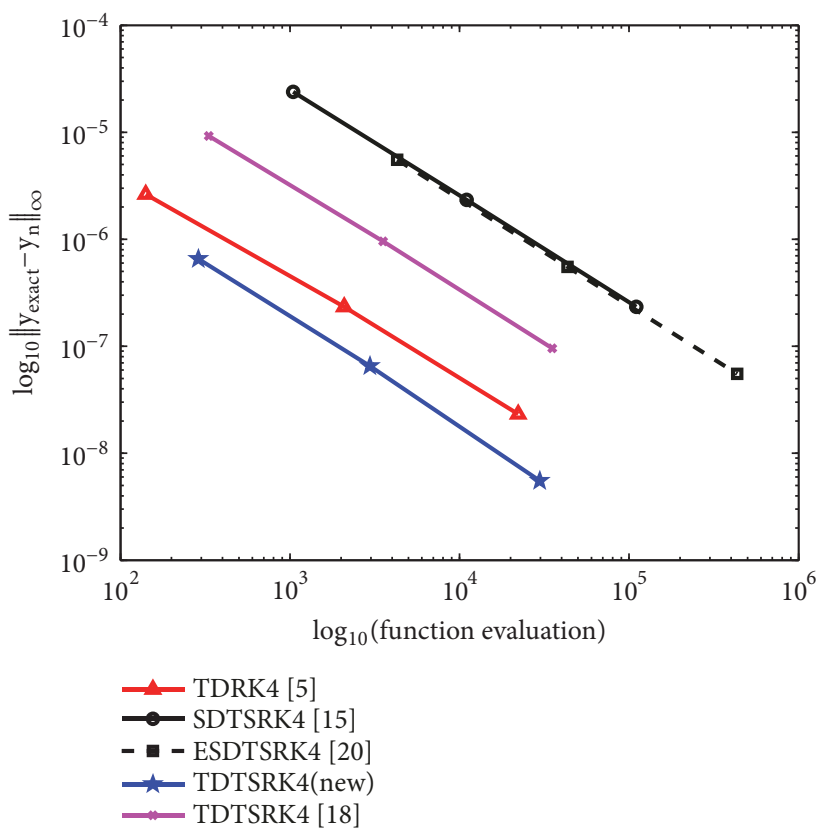

(a)

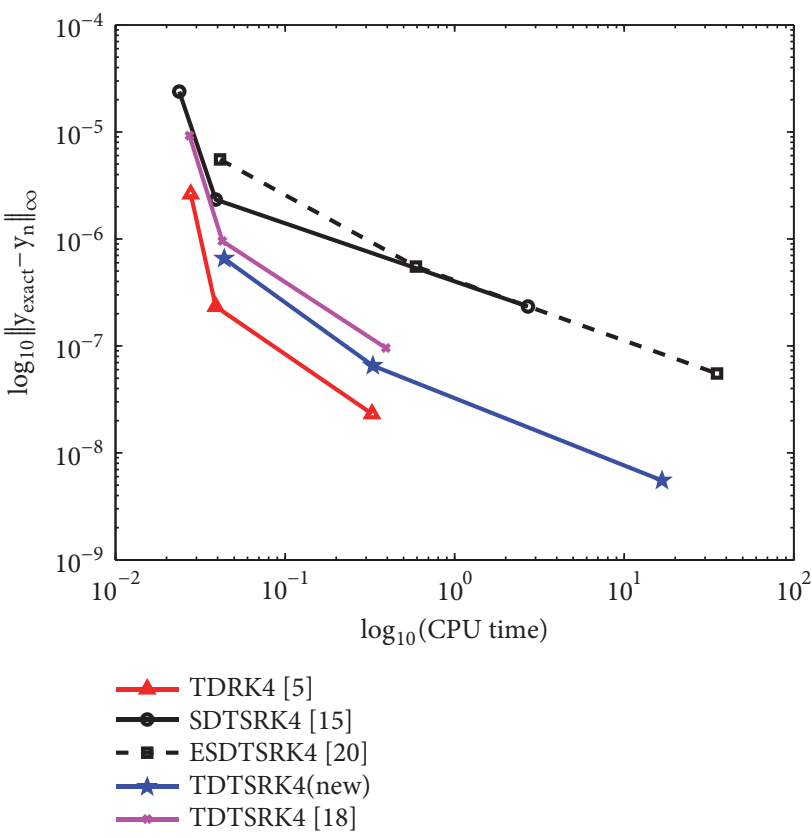

(b)

Figure 2: Efficiency Plots for Problem 1.

the exact solution is known, we have decided to employ the usual test based on computing of the maximum global error over the whole integration interval, because it gives a more significant measure of the efficiency. Figure 2 depicts the efficiency curves for the tested methods. Figures 2(a) and 4(a) show the logarithm of the number of function evaluations against the maximum global error $\left(\log _{10}\left\|y_{\text {exact }}-y_{n}\right\|_{\infty}\right)$, while Figures 2(b) and 4(b) show the logarithm of the maximum global error $\left(\log _{10}\left\|y_{\text {exact }}-y_{n}\right\|_{\infty}\right)$ against the computational effort measured by the CPU time required by each method for Problem 1 and Problem 3.

The plots in Figures 2(a) and 2(b) show that the fourthorder TDTSRK methods compared favorably with other existing methods in $[5,18]$ but outpserform the TDTSRK methods in $[15,20]$ for Problem 1 in terms of accuracy and computational time. We observed that as the value assigned to Tol becomes smaller the accuracy becomes better.

Similarly, Figure 3 depicts the efficiency curves of the tested fourth-order methods on Problem 2. Figure 3(a) shows the logarithm of number of function evaluations against the maximum error $\left(\log _{10}\left\|\bar{y}_{n}-y_{n}\right\|_{\infty}\right)$, while Figure 3(b) depicts the logarithm of the maximum error $\left(\log _{10}\left\|\bar{y}_{n}-y_{n}\right\|_{\infty}\right)$ against the computational effort measured by the CPU time required by each method for Problem 2 for Tol $=10^{-j}, j=1,2,3$. The plots in Figures 3(a) and 3(b) indicate that our fourth-order TDTSRK method compared favorably with the fourth-order 


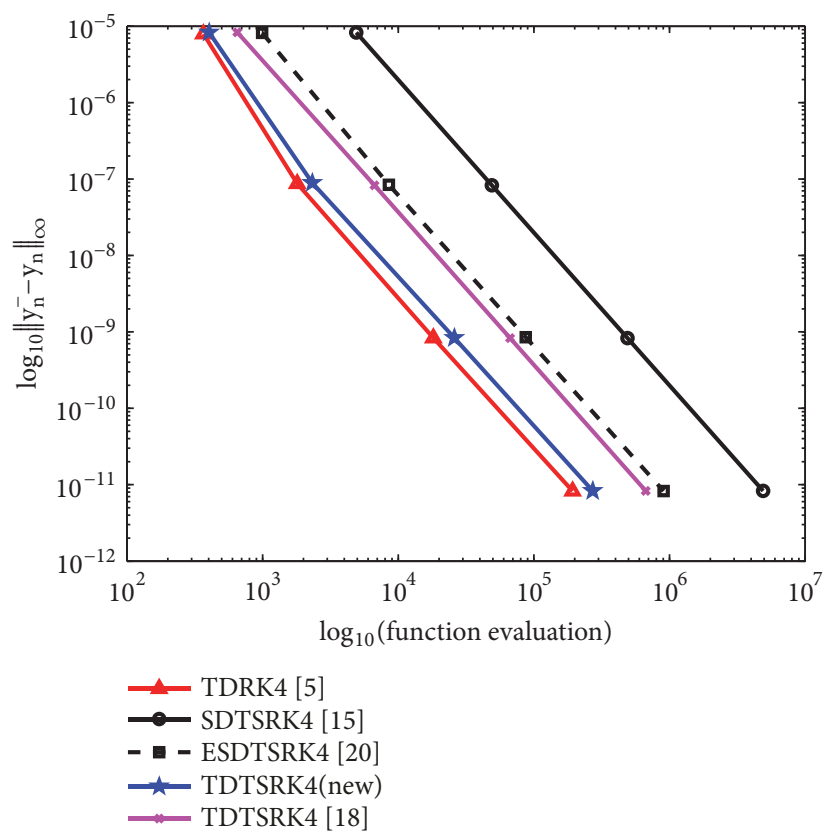

(a)

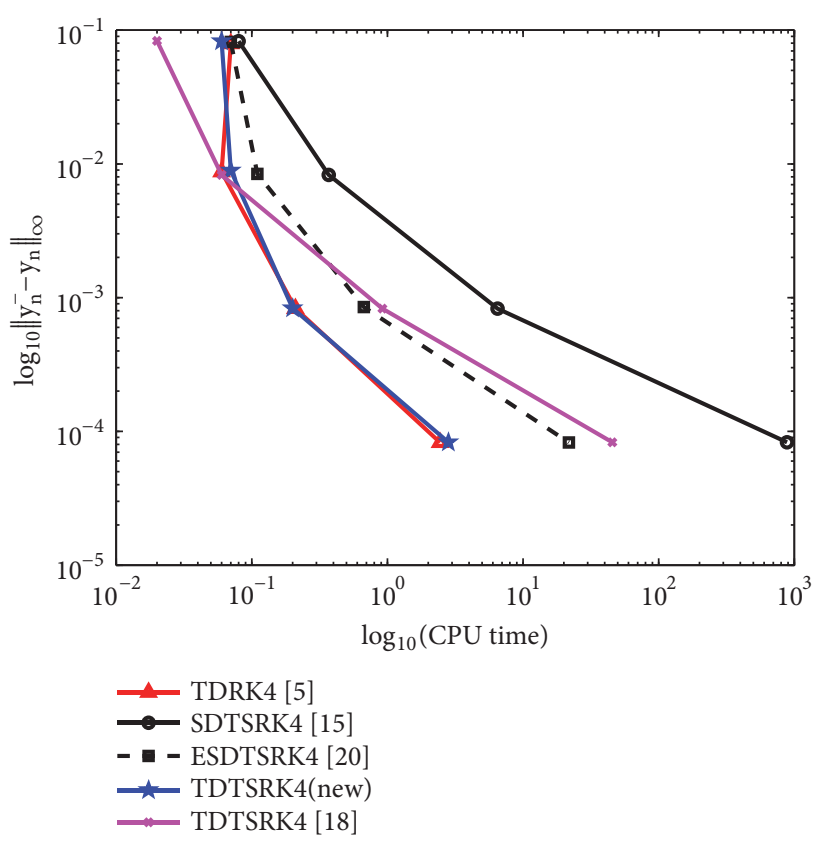

(b)

Figure 3: Efficiency Plots for Problem 2.

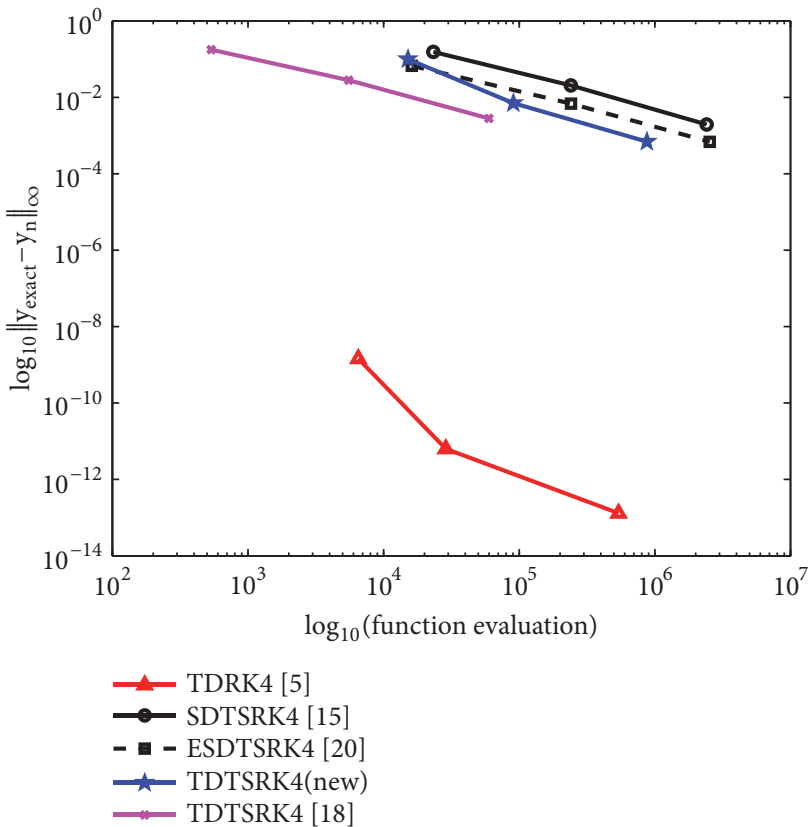

(a)

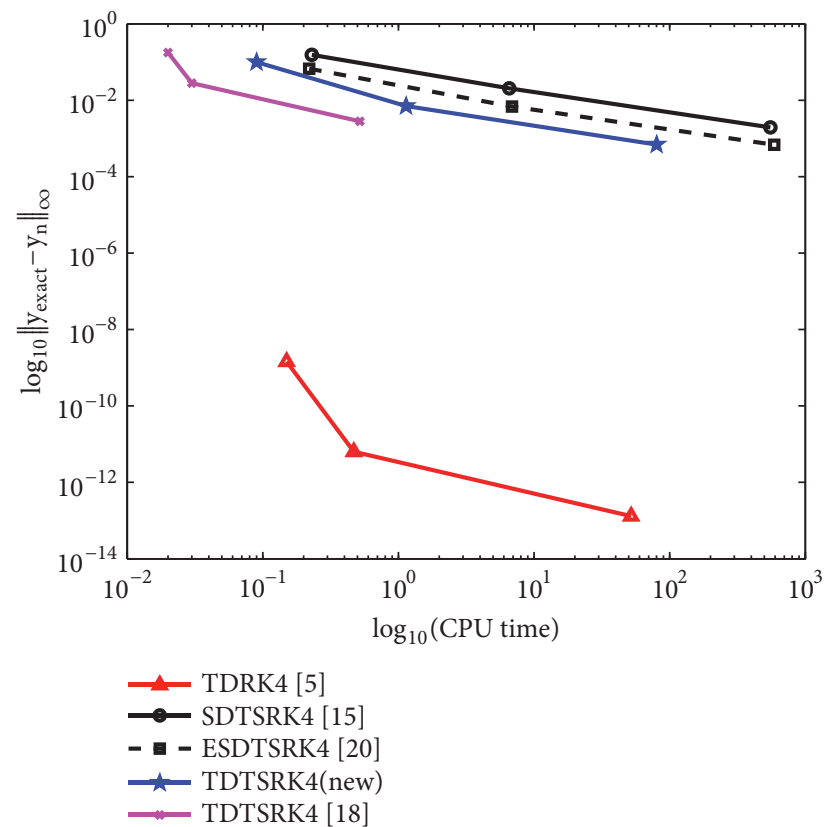

(b)

Figure 4: Efficiency Plots for Problem 3, $\tau=100$.

method in [5], but outperformed the fourth-order TDTSRK methods in $[15,18,20]$ for Problem 2 in terms of accuracy and computational time.

Finally, Figure 4(a) shows the logarithm of number of function evaluations against the maximum norm of error $\left(\log _{10}\left\|y_{\text {exact }}-y_{n}\right\|_{\infty}\right)$, while Figure 4(b) depicts the logarithm of the maximum norm of error $\left(\log _{10}\left\|y_{\text {exact }}-y_{n}\right\|_{\infty}\right)$ against the computational effort measured by the CPU time required by each method for Problem 3 for Tol $=10^{-j}, j=1,2,3$. The plots in Figures 4(a) and 4(b) indicate that our fourth-order TDRK method [5] outperforms the fourth-order methods in $[15,18,20]$ for Problem 3 in terms of accuracy and 
computational time, but the new TDTSRK method is better than the fourth-order TDTSRK methods in $[15,18,20]$ for Problem 3 in terms of accuracy and computational time.

\section{Conclusion}

We have developed and implemented our methods alongside other well-known schemes of the same order as the schemes we developed. Some initial value problems (IVPs) were used to test the efficiency of our methods. The accuracy of our method can be shown from the numerical examples.

\section{Appendix}

The coefficients of TDTSRK method of order $p=6, s=3$ and $\bar{A}=0$

$$
\begin{aligned}
& b_{11}=-5 b_{13}-24 \bar{b}_{13}+u_{1}, \\
& \bar{b}_{11}=-b_{13}-5 \bar{b}_{13}+\frac{u_{1}}{6}, \\
& b_{12}=4\left(b_{13}+6 \bar{b}_{13}\right), \\
& \bar{b}_{12}=\frac{1}{3}\left(-6 b_{13}-24 \bar{b}_{13}+u_{1}\right), \\
& b_{21}=\frac{1}{4}\left(29-20 a_{21}-20 b_{23}-96 \bar{b}_{23}+4 u_{2}\right), \\
& b_{22}=\frac{1}{4}\left(-27+16 a_{21}+16 b_{23}+96 \bar{b}_{23}\right), \\
& \bar{b}_{21}=\frac{73}{48}-a_{21}-b_{23}-5 \bar{b}_{23}+\frac{u_{2}}{6}, \\
& \bar{b}_{22}=\frac{1}{48}\left(119-96 a_{21}-96 b_{23}-384 \bar{b}_{23}+16 u_{2}\right), \\
& w_{2}=\frac{1}{15}\left(248-720 \bar{v}_{2}-2880 \bar{v}_{3}-135 v_{2}-960 v_{3}\right), \\
& b_{31}=33-5 a_{31}-28 a_{32}-5 b_{33}-24 \bar{b}_{33}+u_{3}, \\
& b_{32}=-32+4 a_{31}+27 a_{32}+4 b_{33}+24 \bar{b}_{33}, \\
& \bar{b}_{31}=\frac{43}{6}-a_{31}-6 a_{32}-b_{33}-5 \bar{b}_{33}+\frac{u_{3}}{6}, \\
&\left.-3510 v_{3}\right), \\
& \bar{b}_{32}=\frac{1}{3}\left(757-1980 \bar{v}_{2}-13320 \bar{v}_{3}-300 v_{2}\right. \\
&\left.\bar{b}_{32}-6 b_{33}-24 \bar{b}_{33}+u_{3}\right) \\
& \bar{b}_{3}+v_{1}+18 v_{2}+180 v_{3},
\end{aligned}
$$

$$
\begin{aligned}
\bar{w}_{1} & =\frac{1}{20}\left(77-200 \bar{v}_{2}-1380 \bar{v}_{3}-30 v_{2}-360 v_{3}\right), \\
\bar{w}_{2} & =\frac{1}{3}\left(64-171 \bar{v}_{2}-1056 \bar{v}_{3}-27 v_{2}-288 v_{3}\right), \\
\bar{w}_{3} & =\frac{1}{60}\left(529-60 \bar{v}_{1}-1440 \bar{v}_{2}-7200 \bar{v}_{3}-270 v_{2}\right. \\
& \left.-2160 v_{3}\right) .
\end{aligned}
$$

\section{Data Availability}

No data.

\section{Conflicts of Interest}

The authors declare that they have no conflicts of interest.

\section{References}

[1] A. Abdi and G. Hojjati, "Maximal order for second derivative general linear methods with Runge-Kutta stability," Applied Numerical Mathematics, vol. 61, no. 10, pp. 1046-1058, 2011.

[2] Z. Bartoszewski and Z. Jackiewicz, "Construction of two-step Runge-Kutta methods of high order for ordinary differential equations," Numerical Algorithms, vol. 18, no. 1, pp. 51-70, 1998.

[3] J. C. Butcher and S. Tracogna, "Order conditions for two-step Runge-Kutta methods," Applied Numerical Mathematics, vol. 24, no. 2-3, pp. 351-364, 1997.

[4] J. C. Butcher, Numerical Methods for Ordinary Differential Equations, John Wiley \& Sons, Chichester, UK, 3rd edition, 2016.

[5] R. P. K. Chan and A. Y. Tsai, "On explicit two-derivative RungeKutta methods," Numerical Algorithms, vol. 53, no. 2-3, pp. 171194, 2010.

[6] J. Chollom and Z. Jackiewicz, "Construction of two-step RungeKutta methods with large regions of absolute stability," Journal of Computational and Applied Mathematics, vol. 157, no. 1, pp. 125-137, 2003.

[7] R. D’Ambrosio and Z. Jackiewicz, "Continuous two-step RungeKutta methods for ordinary differential equations," Numerical Algorithms, vol. 54, no. 2, pp. 169-193, 2010.

[8] K. H. Kastlunger and G. Wanner, "Runge Kutta processes with multiple nodes," Computing, vol. 9, no. 1, pp. 9-24, 1972.

[9] E. Hairer and G. Wanner, "Order conditions for general twostep Runge-Kutta methods," SIAM Journal on Numerical Analysis, vol. 34, no. 6, pp. 2087-2089, 1997.

[10] Z. Jackiewicz, R. Renaut, and A. Feldstein, “Two-step RungeKutta methods," SIAM Journal on Numerical Analysis, vol. 28, no. 4, pp. 1165-1182, 1991.

[11] Z. Jackiewicz and S. Tracogna, "A general class of two-step Runge-Kutta methods for ordinary differential equations," SIAM Journal on Numerical Analysis, vol. 32, no. 5, pp. 13901427, 1995.

[12] Z. Jackiewicz, R. Renaut, and M. Zennaro, "Explicit two-step Runge-Kutta methods," Applications of Mathematics, vol. 40, no. 6, pp. 433-456, 1995.

[13] Z. Jackiewicz and J. H. Verner, "Derivation and implementation of two-step Runge-Kutta pairs," Japan Journal of Industrial and Applied Mathematics, vol. 19, no. 2, pp. 227-248, 2002. 
[14] R. I. Okuonghae and M. N. O. Ikhile, "Second derivative general linear methods," Numerical Algorithms, vol. 67, no. 3, pp. 637654, 2014.

[15] R. I. Okuonghae and I. B. Aiguobasimwin, "Second derivative two-step Runge-Kutta methods," Journal of the Nigerian Institution of Production Engineering, vol. 21, pp. 2087-2089, 2017.

[16] L. M. Skvortsov, "Explicit two-step Runge-Kutta methods," Mathematical Models and Computer Simulations, vol. 2, no. 2, pp. 222-231, 2010.

[17] S. Tracogna, "Implementation of two-step Runge-Kutta methods for ordinary differential equations," Journal of Computational and Applied Mathematics, vol. 76, no. 1-2, pp. 113-136, 1996.

[18] O. M. Turaci and S. T. Öziş, "On explicit two-derivative two-step RungeKutta methods," Computational and Applied Mathematics, 2018.

[19] Z. Jackiewicz, General Linear Methods for Ordinary Differential Equations, John Wiley \& Sons, Chichester, UK, 2009.

[20] I. B. Aiguobasimwin, Explicit Second derivative two-step RungeKutta methods for non-stiff IVPs. [M.Phil Thesis], Department of Mathematics, University of Benin, Benin City, Nigeria, 2019.

[21] R. L. Burden and J. D. Faires, Numerical Analysis, PWS Publishing Company, 5th edition, 1993. 




Advances in

Operations Research

\section{-n-m}
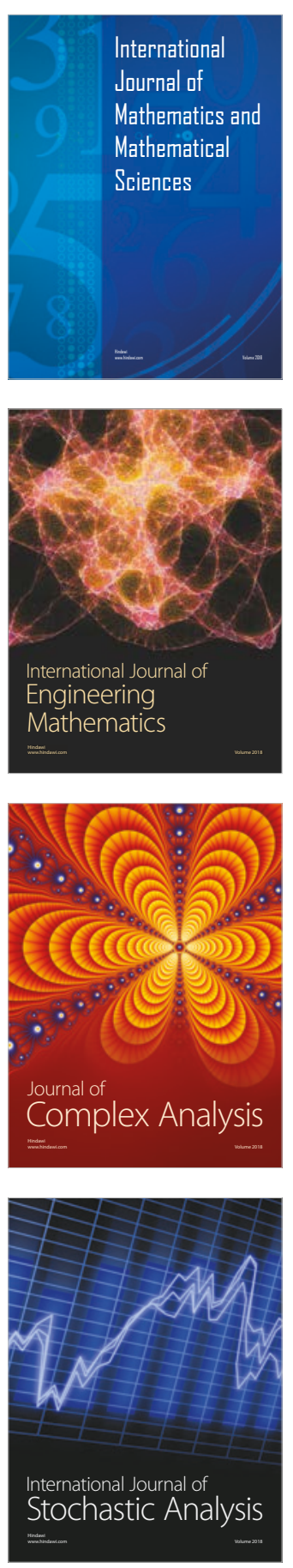
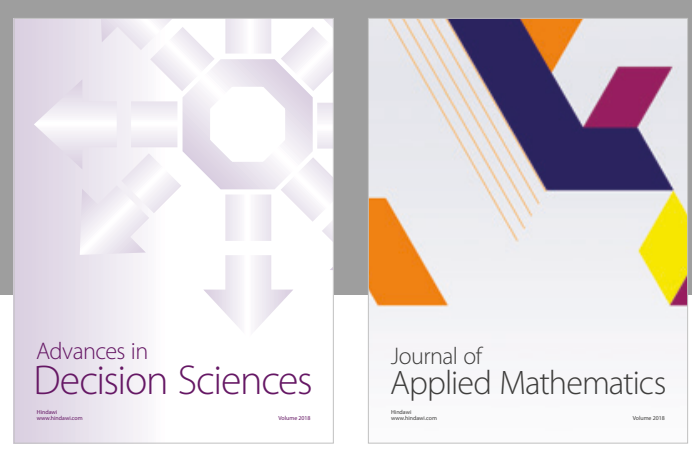

Journal of

Applied Mathematics
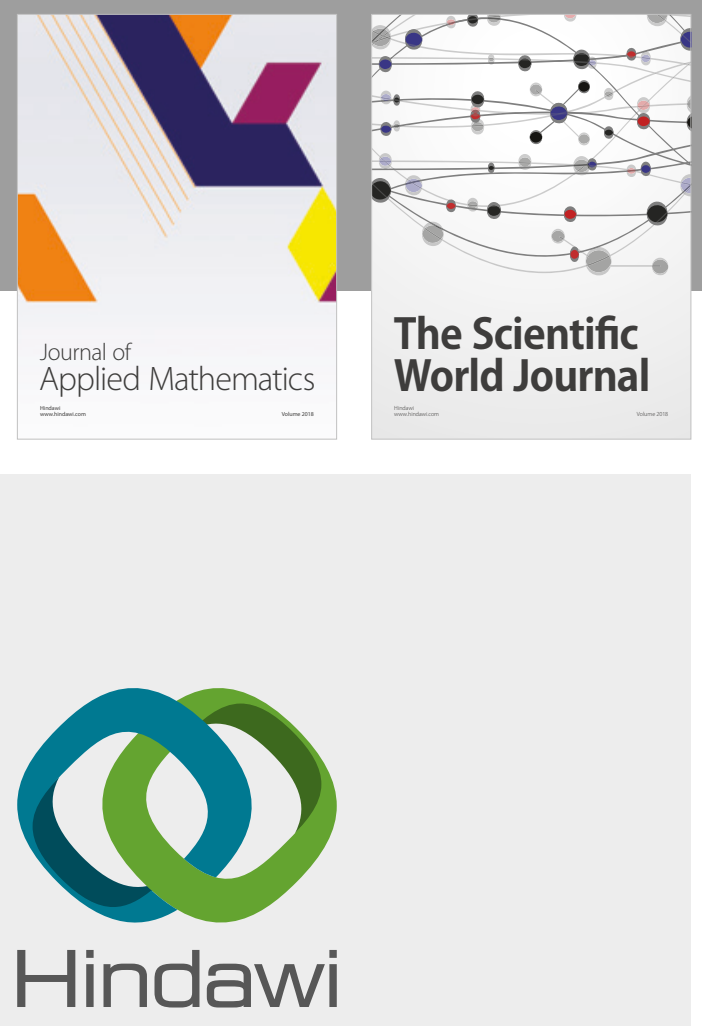

Submit your manuscripts at

www.hindawi.com

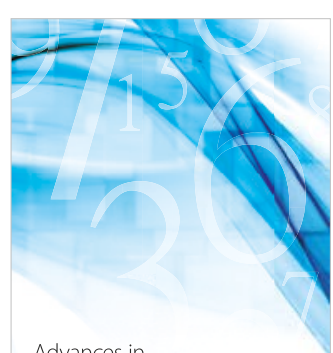

Advances in
Numerical Analysis
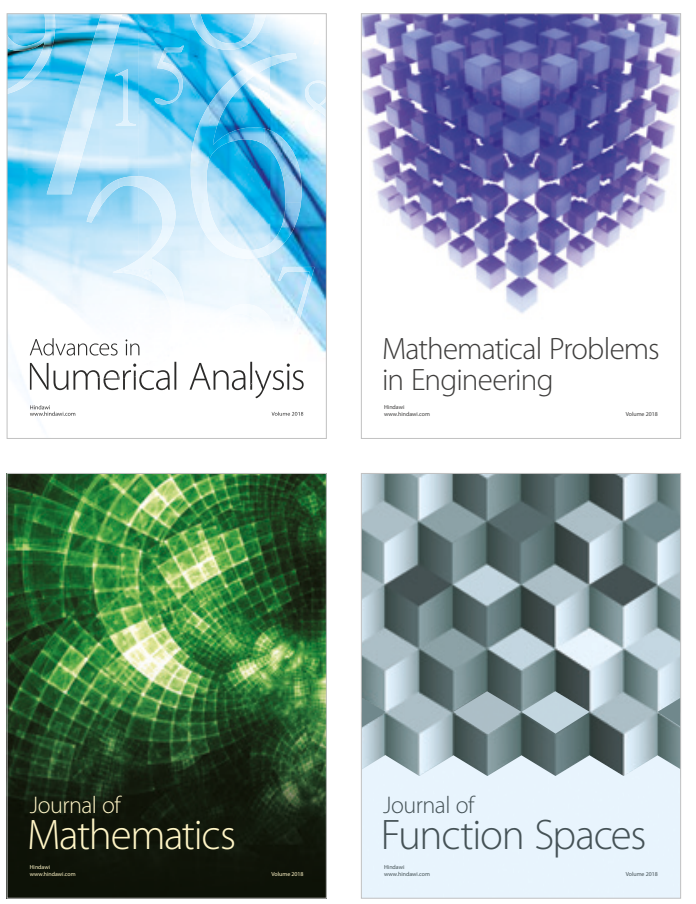

Mathematical Problems in Engineering



International Journal of

Differential Equations

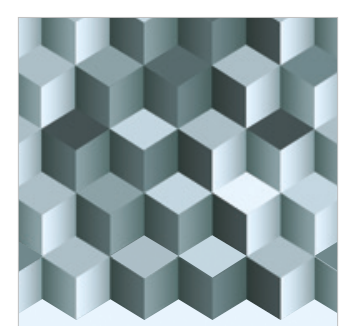

Journal of

Function Spaces

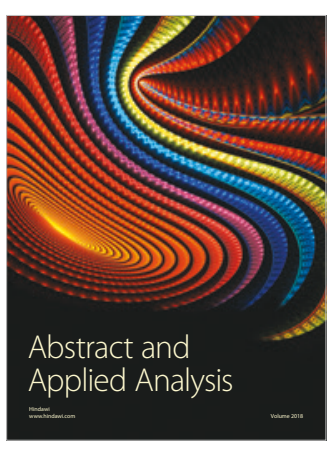

The Scientific

World Journal

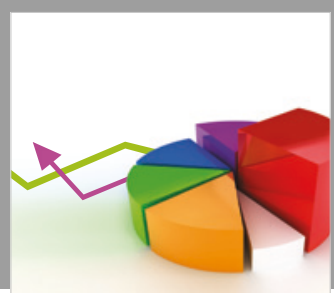

Journal of

Probability and Statistics
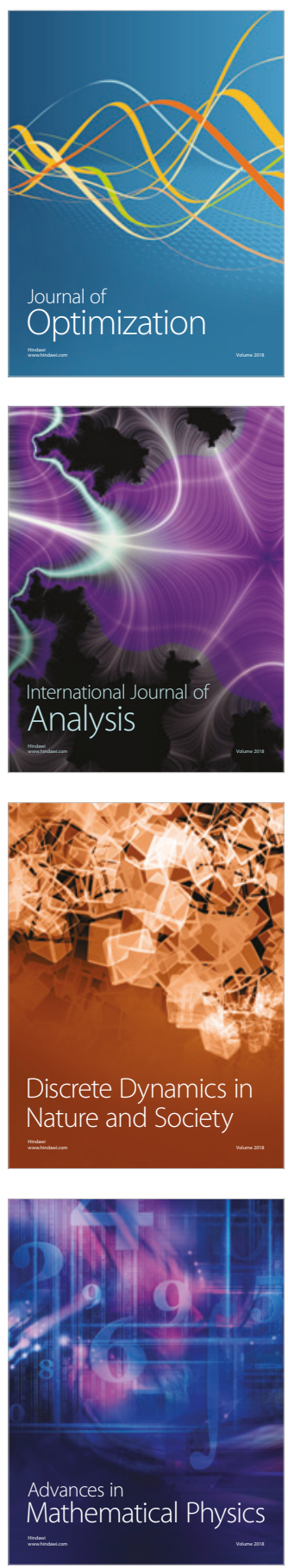